\title{
Fibre prestressed composites: theoretical and numerical modelling of unidirectional and plain-weave fibre reinforcement forms
}

\begin{abstract}
The objective of this study is to analyse the residual stresses induced in fibre prestressed composites. Both theoretical and numerical methods have been included in the analysis. Macro-mechanical approach of fibre reinforced composite has been developed to include the prestressing effect that suitable for analysing both unidirectional and plain-weave fabric composites. A new simplistic theoretical model was also derived to estimate residual stresses in composite constituents due to releasing the fibre pretension load. This model can be successfully used for composites reinforced by unidirectional fibres. Prestressed composites were modelled numerically in order to validate the theoretical results and estimate the full distribution of residual stresses within the composite constituent more precisely. Good agreement has been obtained between theoretical and numerical results. The results obtained in this study clearly showed that the level of induced residual stresses within the composite constituents depends not only on fibre pretention level, but also on the composite elastic properties.
\end{abstract}

Keyword: Fibre prestressed composites; Theoretical analysis; Numerical modelling; Residual stresses; Unidirectional and plain-weave fabric 